\title{
Temperature Variations and Abundance Determinations in Planetary Nebulae
}

Silvia Torres-Peimbert and Manuel Peimbert

Instituto de Astronomía, Universidad Nacional Autónoma de México; Apdo. postal 70-264; Ciudad Universitaria; México D.F. 04510; México.

\begin{abstract}
It is argued that an important fraction of PNe present large temperature variations that are not due to observational errors nor to incomplete atomic physics. Seven possible causes for these variations are reviewed, one of them is presented for the first time.
\end{abstract}

\section{Overview}

Photoionization models for chemically homogeneous gaseous nebulae of constant density predict an almost constant temperature and consequently very often observers assume a constant temperature to determine chemical abundances. There is growing observational evidence that indicates the presence of large temperature variations in gaseous nebulae, in contradiction with the models mentioned above. Consequently the relevance of temperature variations in the abundance determinations has to be considered in detail.

In section 2 we review the observational evidence in favor of large temperature variations. In section 3 we review different possible explanations for the observed variations. In section 4 we discuss the implications of the temperature variations in the abundance determinations, these implications depend on the source of the temperature variations.

Recent reviews on the temperature structure of gaseous nebulae are those of Peimbert, M. (1995, 2002), Esteban (2002), Stasińska (2002), and Liu (2002a, 2002b).

\section{Temperature Variations}

\subsection{Definitions}

The average temperature, $T_{0}$, and the mean square temperature fluctuation, $t^{2}$, are given by

$$
T_{0}\left(N_{e}, N_{i}\right)=\frac{\int T_{e}(\mathbf{r}) N_{e}(\mathbf{r}) N_{i}(\mathbf{r}) d V}{\int N_{e}(\mathbf{r}) N_{i}(\mathbf{r}) d V}
$$

and

$$
t^{2}=\frac{\int\left(T_{e}-T_{0}\right)^{2} N_{e} N_{i} d V}{T_{0}^{2} \int N_{e} N_{i} d V}
$$

respectively, where $N_{e}$ and $N_{i}$ are the electron and the ion densities of the observed emission line and $V$ is the observed volume (Peimbert 1967). 
To determine $T_{0}$ and $t^{2}$ we need two different methods to derive $T_{e}$ : one that weighs preferentially the high temperature regions and one that weighs preferentially the low temperature regions. For example the temperature derived from the ratio of the [O III] $\lambda \lambda 4363,5007$ lines, $T_{(4363 / 5007)}$, and the temperature derived from the ratio of the Balmer continuum to $I(H \beta), T_{(\mathrm{Bac} / H \beta)}$, that are given by

$$
T_{(4363 / 5007)}=T_{0}\left[1+\frac{1}{2}\left(\frac{90800}{T_{0}}-3\right) t^{2}\right]
$$

and

$$
T_{(\mathrm{Bac} / \mathrm{H} \beta)}=T(\mathrm{Bac})=T_{0}\left(1-1.70 t^{2}\right),
$$

respectively. It is also possible to use the intensity ratio of a collisionally excited line of an element $p+1$ times ionized to a recombination line of the same element $p$ times ionized, this ratio is independent of the element abundance and in the low density limit depends only on the electron temperature. Two examples of this method are:

$$
T_{(\mathrm{O} \text { IIrec } / \mathrm{O} \text { IIIcoll })}=T_{(4649 / 5007)}=f_{1}\left(T_{0}, t^{2}\right)
$$

and

$$
T_{(\mathrm{C} \mathrm{IIrec} / \mathrm{C} \mathrm{IIIcoll})}=T_{(4267 / 1909)}=f_{2}\left(T_{0}, t^{2}\right),
$$

by combining these ratios with a temperature determined from the ratio of two collisionally excited lines, like $T_{(4363 / 5007)}$, it is also possible to derive $T_{0}$ and $t^{2}$.

From the ratio of two He I recombination lines it is possible to obtain relationships of the type:

$$
T_{(\mathrm{He} \mathrm{I}, i / j)}=f_{i j}\left(T_{0}, t^{2}, N_{e}, \tau_{3889}\right),
$$

and from a set of 5 or more helium lines (which should include $\lambda \lambda 3889,6678$, and 7065) it is possible to obtain $T_{0}, t^{2}, N_{e}$, and $\tau_{3889}$ (see Peimbert, Peimbert, $\&$ Ruiz 2000). Sometimes the errors in these determinations are quite large, in such case it is possible to combine the information from the helium lines with the one from $T_{(4363 / 5007)}$ to obtain better determinations of $T_{0}$ and $t^{2}$ (see Peimbert, Peimbert, \& Luridiana 2002).

\subsection{Errors in the temperature determinations}

Different types of errors are present in the determinations of the electron temperatures, and in some cases these errors have led to erroneous temperature determinations. Before accepting that temperature variations are present in a given object, it is necessary to rule out any possible sources of error.

Rola \& Stasińska (1994) and Rola \& Pelat (1994) have argued that there is a systematic overestimation of the line intensities of lines with low signal to noise ratios that leads to the overestimation of the $T_{(4267 / 1909)}$ temperatures. This effect is present in many objects and has to be taken into consideration, but for many well observed objects it is not the main cause of the derived large $T_{(4363 / 5007)}-T_{(4267 / 1909)}$ differences (e.g., Peimbert, Torres-Peimbert, \& Luridiana 1995).

The discrepancy between the C abundances derived from the C II $\lambda 4267$ recombination line and those derived from the collisionally excited 1906+1909 
C III lines has been known for at least 20 years and the idea that the large intensity of the C II $\lambda 4267$ line was due to an incomplete knowledge of the relevant atomic physics has been mentioned on several occasions. Nevertheless similar excesses in the intensities of recombination lines are observed in different multiplets of the same ion and in other elements (Liu et al. 1995; Mathis, Torres-Peimbert, \& Peimbert 1998).

Viegas \& Clegg (1994) suggested that the differences between $T_{(4649 / 5007)}$ and $T(\mathrm{Bac})$ could be due to density fluctuations diminishing the $I(4959)$ and $I(5007)$ values by collisional de-excitation yielding a spuriously high $T_{(4649 / 5007)}$ value. This is indeed the case for the planetary nebula M2-29, where from ground based observations of the whole object a $T_{(4363 / 5007)}$ of $24,000 \mathrm{~K}$ and a $N_{e}=2000 \mathrm{~cm}^{-3}$ are derived, while from HST observations it is found that it has at least two density components (one with $N_{e} \sim 10^{4} \mathrm{~cm}^{-3}$ and another with $N_{e} \sim 10^{6} \mathrm{~cm}^{-3}$, the $T_{e}$ in the low density component is of $8800 \mathrm{~K}$ ); the high temperature derived from the ground observations is spurious and yields $\mathrm{O} / \mathrm{H}=5.1 \times 10^{-5}$, a factor of ten smaller than the value derived adopting a two density component model (Peña, Torres-Peimbert, \& Ruiz 1991; TorresPeimbert et al. 2002). A similar result has been found for $\mathrm{Mz} 3$ by Zhang \& Liu (2002).

\subsection{Observations of Temperature Variations}

From uniform density chemically homogeneous photoionization models it has been found that $t^{2}$ is in the 0.000 to 0.025 range, with typical values around 0.005 (e.g., Gruenwald \& Viegas 1995).

From observations and by combining equation 3 with any of the equations 4 to 7 it is possible to determine $t^{2}$ and $T_{0}$. These combinations indicate values of $t^{2}$ in the 0.00 to 0.15 range with typical values around 0.04 . The errors in the best $t^{2}$ determinations given in the literature are typically in the 0.01 to 0.02 range.

Some of the best determinations of $t^{2}$ and $T_{0}$ are: from equations 3 and 4 those by Peimbert (1971), Liu \& Danziger (1993), Liu et al. (1995, 2000, 2001); from equations 3 and 5 those by Peimbert, Storey, \& Torres-Peimbert (1993), Liu et al. (1995, 2000, 2001); from equations 3 and 6 those by Rola \& Stasińska (1994), Peimbert, et al. (1995); and from equations 3 and 7 those by Peimbert et al. $(1995,2000,2002)$.

Liu et al. (2001, see also Peimbert, A. 2002), presented a good correlation between $\log \left(\mathrm{O}^{++} / \mathrm{H}^{+}\right)_{R L} /\left(\mathrm{O}^{++} / \mathrm{H}^{+}\right)_{C E L}$ and $T_{(4363 / 5007)}-T(\mathrm{Bac})$ and mention that this correlation strongly supports the idea that the temperature variations are real. In Table 1 we present the $T_{(4363 / 5007)}$ and $T(\mathrm{Bac})$ temperatures derived by Liu et al. $(2000,2001)$ for three of the objects that show very large temperature variations. From these values we have computed $T_{(4649 / 5007)}$ and from equations 3 and 4 we have derived $t^{2}(3,4)$ and from equations 3 and 5 we have derived $t^{2}(3,5)$. From this table it can be seen that $t^{2}(3,4)$ and $t^{2}(3,5)$ are not only proportional to each other but that they are in good agreement with each other. This implies that most of the temperature differences among $T(\mathrm{Bac}), T_{(4363 / 5007)}$, and $T_{(4649 / 5007)}$ are due to real temperature variations in the nebulae, thus an explanation for the origin of these variations has to be sought. 
Table 1

Mean Square Temperature Fluctuation Values

\begin{tabular}{lccccc}
\hline \hline Object & $T_{(4363 / 5007)}$ & $T_{(\mathrm{Bac} / \mathrm{H} \beta)}$ & $T_{(4649 / 5007)}$ & $t^{2}(3,4)$ & $t^{2}(3,5)$ \\
\hline M1-42 & 9220 & 3560 & 5550 & 0.123 & 0.120 \\
M2-36 & 8380 & 5900 & 6025 & 0.053 & 0.078 \\
NGC 6153 & 9140 & 6080 & 6220 & 0.065 & 0.097 \\
& & & & & \\
\hline \hline
\end{tabular}

\section{What Causes the Temperature Variations?}

In what follows we present seven different mechanisms that can produce large temperature variations. It is clear that all of them should be considered. Their relative importance probably varies for each object, and in many cases, the ones that show $t^{2}<0.01$ values, all of them are negligible.

\subsection{Shadowed Regions}

To explain the intensities of lines of low degree of ionization Mathis (1976) suggested the existence of regions shadowed from the central stars, these regions would be ionized by diffuse radiation and would have a considerably smaller temperature than those regions ionized directly from the central stars of $\mathrm{H} \mathrm{II}$ regions.

\subsection{Chemical Inhomogeneities}

Some PNe, like A30 and A78, present important chemical inhomogeneities; therefore the idea that temperature variations can be due to various degrees of chemical inhomogeneities has been proposed. Chemically inhomogeneous photoionization models have been presented by different authors (e.g., TorresPeimbert, Peimbert, \& Peña 1990; Kingdon \& Ferland 1998; Pequignot et al. 2002a, 2002b; Tylenda 2002).

\subsection{Density Variations}

Extreme density variations are present in most $\mathrm{PNe}$, as can be seen from optical images; these clumps, shells, and filaments, can produce temperature variations by themselves under the assumption of photoionization heating only. Mihalszky \& Ferland (1983) have presented chemically homogeneous photoionization models, including density variations in the $10^{2}$ to $10^{4.3} \mathrm{~cm}^{-3}$ range, and find values of $t^{2}$ of about 0.005 , which could explain only a small fraction of the largest discrepancies observed.

\subsection{Deposition of Mechanical Energy}

Shocks have been proposed as a mechanism to account for temperature fluctuations (Peimbert, Sarmiento, \& Fierro 1991; Peimbert et al. 1995 and references therein). Peimbert et al. (1995) have found that those objects that show higher 
velocity dispersions are the ones that show higher temperature variations, supporting the presence of shocks. In a PN a shock front is expected to be formed at the interface between a fast stellar wind from the central star and the slowly expanding circumstellar envelope. The fast stellar wind would carve out a hot bubble in the interior of the nebula that would emit primarily in X-rays. Clear evidence for such hot bubbles has been found recently for a small number of PNe from X-ray imaging (Kastner et al. 2000; Kastner, Vrtilek, \& Soker 2001; Chu et al. 2001).

Mellema (1997) finds strong temperature variations from hydrodynamical simulations of bipolar PNe. The dense gas near the equator has temperatures around $9000 \mathrm{~K}$ while the gas near the poles has temperatures between 20,000 and $50,000 \mathrm{~K}$. The reasons are two: at the poles the nebula has a higher shock velocity, leading to more heating; and a lower density, leading to less efficient cooling. Bohigas (2002) finds that in the outer regions of 9 PNe of Type I the $T\left(\mathrm{O}^{++}\right) / T\left(\mathrm{~N}^{+}\right)$is large and the $\mathrm{H} \alpha /[\mathrm{S} \mathrm{II}]$ line ratio is small, a typical combination in shock excited plasmas.

\subsection{Deposition of Magnetic Energy}

Ferland, in the meeting on Ionized Gaseous Nebulae (Mexico City 2000), suggested that magnetic reconnection can provide localized temperature variations in ionized plasmas. According to García-Segura, López, \& Franco (2001) the multiple, regularly spaced concentric shells around some PNe could be due to the effects a of a solar-like magnetic cycle, with periodic polarity inversion, in the slow wind of an asymptotic giant branch (AGB) star. Presumably these shells of alternating polarity could give rise to magnetic reconnection processes once that they are compressed in the formed PN (i.e. in the swept-up shell). Magnetic fields of the order of milligauss have been measured in the torus surrounding K3-35 (Miranda et al. 2001); also the presence of a magnetic field has been inferred in the planetary nebula OH0.9+1.3 (Zijlstra et al. 1989) and in the pre-planetary nebula IRAS17150-3224 (Hu et al. 1993).

\subsection{Dust Heating}

Stasińska \& Szczerba (2001) have analyzed the effects of photoelectric heating by dust grains in photoionization models of PNe. They have shown that this process is particularly important if filamentary PNe contain a population of small grains. The temperature structure of such dusty and filamentary nebulae would produce large temperature variations.

\subsection{Decrease of the Ionizing Flux}

The ionizing flux from the central stars of $\mathrm{PNe}$ is not constant. As a first approximation the ionization increases, then reaches a maximum and finally decreases. During the decrease and in those directions where the nebula is density bounded there would be ionized regions that would become isolated from the stellar ionizing photons, these regions would cool first and would recombine later (e.g., Spitzer 1978). A typical recombination time is $\sim 10^{5} / N_{e}$ years while the cooling time is an order of magnitude smaller, therefore cool regions with a relatively high degree of ionization are expected to be present. 
This effect might be important for PNe produced by massive stars, because the central stars evolve relatively fast. One possible example of this mechanism might be provided by $\mathrm{N} 66$ in the LMC. In this object the ionizing flux has diminished by a factor of two in the last ten years (Peña et al. 1997; Peña, Hamann, \& Ruiz 2002) and the RL/CEL abundance ratio is of about an order of magnitude (Tsamis et al. 2002). Other PNe central stars have presented important flux changes in timescales of a few years (e.g., Kostiakova 2002 and references therein). It is possible that this mechanism might account for the very low values of $T(\mathrm{Bac}), \sim 3000 \mathrm{~K}$, found by Luo \& Liu (2002) in the outer parts of NGC 7009.

It would be possible also to isolate regions from the stellar ionizing photons if the amount of material in the line of sight increases with time, even if the stellar ionizing radiation remains constant. It has been suggested that dust clouds moving tangentially to the line of sight are responsible for the 1981-1985 and 1996-1997 fadings of V651 Mon, the central star of NGC 2346 (Costero et al. 1986; Kato, Nogami, \& Baba 2001). In addition to dust clouds a similar effect would be caused by tangentially moving gaseous clouds of higher density than the ambient density.

\section{Discussion}

Temperature variations higher than those predicted by chemically homogeneous photoionization models of constant density are definitively present in a good fraction of PNe.

Chemically homogeneous photoionization models of constant density provide a good first approximation to the temperature structure and to the chemical abundances only for about half of the well observed nebulae. However, for the other half, it is paramount to have observations of high accuracy to determine their temperature structure which is needed to obtain accurate abundances of heavy elements relative to hydrogen.

Spatially resolved values of $T_{0}$ and $t^{2}$ are needed to decide among the various possibilities mentioned above. For example, if $T_{0}$ decreases in the outer regions, the temperature variations might be due to a decrease of the ionizing flux; in the opposite case the temperature variations could be due to shock heating.

A very important observational clue to study this problem is provided by Garnett \& Dinerstein (2001) who find a large range in the difference between the $\mathrm{O}^{++}$abundances derived from O II and those derived from [O III], spanning from no difference up to a factor of six. The size of this discrepancy is anti-correlated with nebular surface brightness; compact, high-surface-brightness nebulae have the smallest discrepancies.

It is important to determine the cause of the temperature variations to be able to constrain the models of stellar evolution and of galactic chemical evolution. If the temperature fluctuations due to chemical inhomogeneities dominate, the proper abundances to use are those derived from forbidden lines under the assumption that $t^{2} \sim 0.01$; alternatively if the temperature fluctuations due to the combination of the other six mechanisms mentioned above dominate, the proper abundances to use are those provided by the recombination lines. 
The $\mathrm{C} / \mathrm{H}$ values derived from recombination lines in $\mathrm{PNe}$ are in better agreement with chemical evolution models of the Galaxy than the values derived from collisionally excited lines under the assumption that $t^{2}=0.000$ (Carigi 2002). Alternatively the abundances derived from the infrared lines seem to be in favor of chemical inhomogeneities, but these abundances could be higher if large density fluctuations are present.

The presence of abundance gradients in the Galaxy and the values of $\Delta Y / \Delta Z$ and $\Delta Y / \Delta O$ derived from galactic and extragalactic $H$ II regions indicate that there are significant temperature variations in $\mathrm{H}$ II regions and that they are not due to chemical inhomogeneities (see Peimbert \& Peimbert 2002 for a review).

Acknowledgments. We are gratefull to G. García-Segura, A. Peimbert, and M. Peña, for several excellent suggestions, and to our Australian colleagues for an outstanding meeting.

\section{References}

Bohigas, J. 2002, these proceedings

Carigi, L. 2002, in Ionized Gaseous Nebulae, RMexAACS, 12, 232

Chu, Y.-H., Guerrero, M. A., Gruendl, R. A., Williams, R. M., \& Kaler, J. B. 2001, ApJ, 553, L69

Costero, R., Tapia, M., Echevarría, J., Roth, M., Quintero, A., \& Barral, J. F. 1986, RMexAA, 13, 149

Esteban, C. 2002, in Ionized Gaseous Nebulae, RMexAACS, 12, 56

García-Segura, G., López, J. A., \& Franco, J. 2001, ApJ, 560, 928

Garnett, D. R., \& Dinerstein, H. L. 2001, RMexAASC, 10, 13

Gruenwald, R., \& Viegas, S.M. 1995, A\&A, 303, 535

Hu, J. Y., Slijkhuis, S., Rieu, N.-Q., \& de Jong, T. 1993, A\&A, 273, 185

Kastner, J. H., Soker, N., Vrtilek, S. D., \& Dgani, R. 2000, ApJ, 545, L57

Kastner, J. H., Vrtilek, S. D., \& Soker, N. 2001, ApJ, 550, L189

Kato, T., Nogami, D., \& Baba, H. 2001, PASJ, 53, 901

Kingdon, J. B., \& Ferland, G. J. 1998, ApJ, 506, 323

Kostiakova, E. B. 2002, in Ionized Gaseous Nebulae, RMexAACS, 12, 167

Liu, X.-W. 2002a, in Ionized Gaseous Nebulae, RMexAACS, 12, 70

Liu, X.-W. 2002b, these proceedings

Liu, X.-W., \& Danziger, I. J. 1993, MNRAS, 263, 256

Liu, X.-W., Luo, S.-G., Barlow, M. J., Danziger, I. J., \& Storey, P. J. 2001, MNRAS, 327,141

Liu, X.-W., Storey, P. J., Barlow, M. J., \& Clegg, R. E. S. 1995, MNRAS, 272, 369

Liu, X.-W., Storey, P. J., Barlow, M. J., Danziger, I. J., Cohen, M., \& Bryce, M. 2000, MNRAS, 312,585

Luo, S.-G., \& Liu, X.-W 2002, these proceedings

Mathis, J. S. 1976, ApJ, 207, 442

Mathis, J. S., Torres-Peimbert, S., \& Peimbert, M. 1998, ApJ, 495, 328

Mellema, G. 1997, in IAU Symposium 180, eds. H. J. Habing \& H. J. G. L. M. Lamers, p. 262

Mihalszky, J. S., \& Ferland, J. 1983, PASP, 95, 284

Miranda, L. F., Gómez, Y., Anglada, G., \& Torrelles, J. M. 2001, Nature, 414, 284 
Peimbert, A. 2002, in preparation

Peimbert, A., Peimbert, M., \& Luridiana, V. 2002, ApJ, 565, 668

Peimbert, M. 1967, ApJ, 150, 825

Peimbert, M. 1971, Bol. Obs. Tonantzintla y Tacubaya, 6, 29

Peimbert, M. 1995, in The Analysis of Emission Lines, eds. R. E. Williams, \& M. Livio (Cambridge), 165

Peimbert, M. 2002, in Ionized Gaseous Nebulae, RMexAACS, 12, 273

Peimbert, M., \& Peimbert, A. 2002, RMexAASC, in press

Peimbert, M., Peimbert, A., \& Ruiz, M. T. 2000, ApJ, 541, 688

Peimbert, M., Sarmiento , A., \& Fierro, J. 1991, PASP, 103, 815

Peimbert, M., Storey , P. J., \& Torres-Peimbert, S. 1993, ApJ, 414, 626

Peimbert, M., Torres-Peimbert, S., \& Luridiana, V. 1995, RMexAA, 31, 131

Peña, M., Hamann, W.-R., Koesterke, L., Maza, J., Méndez, R. H., Peimbert, M., Ruiz, M. T., \& Torres-Peimbert, S. 1997, ApJ, 491, 233

Peña, M., Hamann, W.-R., \& Ruiz, M. T. 2002, these proceedings

Peña, M., Torres-Peimbert, S., \& Ruiz, M. T. 1991, PASP, 103, 865

Pequignot, D., Amara, M., Liu, X.-W., Barlow, M. J., Storey, P. J., Morisset, C., TorresPeimbert, S., \& Peimbert, M. 2002a, in Ionized Gaseous Nebulae, RMexAASC, 12,142

Pequignot, D., Liu, X.-W., Barlow, M. J., Storey P. J., \& Morisset, C. 2002b, these proceedings

Rola, C., \& Pelat, D. 1994, A\&A, 287, 676

Rola, C., \& Stasińska, G. 1994, A\&A, 282, 199

Spitzer L. Jr. 1978, in Physical Processes in the Interstellar Medium (New York: WileyInterscience), p. 139

Stasińska, G. 2002, in Ionized Gaseous Nebulae, RMexAACS, 12, 62

Stasińska, G., \& Szczerba, R. 2001, A\&A, 379, 1024

Torres-Peimbert, S., Dufour, R. J., Peimbert, M., \& Peña, M. 2002, in preparation

Torres-Peimbert, S., Peimbert, M., \& Peña, M.: 1990, A\&A, 233, 540

Tsamis, Y. G., Barlow, M. J., Liu, X.-W., \& Danziger, I. J. 2002, these proceedings

Tylenda, R. 2002, these proceedings

Viegas, S. M., \& Clegg, R. E. S. 1994, MNRAS, 271, 993

Zhang, Y., \& Liu, X.-W. 2002, these proceedings

Zijlstra, A. A., Te Lintel Hekkert, P., Pottasch, S. R., Caswell, J. L., Ratag, M., \& Habing, H. J. 1989 A\&A, 217, 157 